\title{
Probing the quartic Higgs boson self-interaction
}

\author{
Tao Liu, ${ }^{1, *}$ Kun-Feng Lyu, ${ }^{1, \dagger}$ Jing Ren, ${ }^{2,3, *}$ and Hua Xing $\mathrm{Zhu}^{4, \S}$ \\ ${ }^{1}$ Department of Physics, The Hong Kong University of Science and Technology, \\ Hong Kong SAR, People's Republic of China \\ ${ }^{2}$ Department of Physics, University of Toronto, Toronto, Ontario M5S1A7, Canada \\ ${ }^{3}$ Institute for High Energy Physics, Chinese Academy of Sciences, \\ Beijing 100049, People's Republic of China \\ ${ }^{4}$ Department of Physics, Zhejiang University, Hangzhou 310027, People's Republic of China
}

(Received 4 June 2018; published 12 November 2018)

\begin{abstract}
The Higgs self-interactions play a crucial role in exploring the underlying mechanisms of electroweak symmetry breaking and the nature of the phase transition involved. In this article, we propose to probe the quartic Higgs self-interaction at lepton and hadron colliders, via the di-Higgs productions. We analyze the contributions of the quartic Higgs coupling, including the renormalization of the cubic Higgs coupling and the modification of the $V V h h$ form factor, to the vector boson-fusion and the vector boson-associated diHiggs productions at one-loop level. Such an effect is independent of the choice of gauge fixing, if the quartic Higgs coupling is decoupled from other couplings in the contexts considered. Notably, a combination of these two di-Higgs productions is important for optimizing the collider sensitivities to probe the quartic Higgs coupling. With this guideline, we explore the International Linear Collider and Compact Linear Collider sensitivities, and find that the International Linear Collider has the potential to measure the quartic Higgs coupling, normalized by its standard model value, with a precision of $\sim \pm 25$ $\left(500 \mathrm{GeV}, 4 \mathrm{ab}^{-1}+1 \mathrm{TeV}, 2.5 \mathrm{ab}^{-1}\right)$ and $\sim \pm 20\left(500 \mathrm{GeV}, 4 \mathrm{ab}^{-1}+1 \mathrm{TeV}, 8 \mathrm{ab}^{-1}\right)$, at $1 \sigma$ C.L., after marginalizing the cubic Higgs coupling in the $\chi^{2}$ analysis. The dependence on the renormalization scheme of the cubic Higgs coupling is discussed.
\end{abstract}

DOI: 10.1103/PhysRevD.98.093004

\section{INTRODUCTION}

The Higgs self-interaction is one of the most important targets for experimentalists to measure at colliders. In the standard model $(\mathrm{SM})$, the Higgs potential $V_{\mathrm{SM}}=$ $-\mu^{2} H^{\dagger} H+\lambda\left(H^{\dagger} H\right)^{2}$ is fully determined by the electroweak scale $v=246 \mathrm{GeV}$ and the Higgs mass $m_{h}=125 \mathrm{GeV}$, with $\lambda=m_{h}^{2} / 2 v^{2}$ and $\mu^{2}=m_{h}^{2} / 2$. The cubic and quartic Higgs couplings are then completely fixed,

$$
\lambda_{3, \mathrm{SM}}=\frac{3 m_{h}^{2}}{v}, \quad \lambda_{4, \mathrm{SM}}=\frac{3 m_{h}^{2}}{v^{2}} .
$$

For many reasons, new physics may enter the Higgs potential, driving the electroweak phase transition (EWPT) and

\footnotetext{
*taoliu@ust.hk

†klyuaa@connect.ust.hk

\#jren@physics.utoronto.ca

${ }^{\S}$ zhuhx@zju.edu.cn
}

Published by the American Physical Society under the terms of the Creative Commons Attribution 4.0 International license. Further distribution of this work must maintain attribution to the author(s) and the published article's title, journal citation, and DOI. Funded by SCOAP . yielding a deviation of the Higgs self-couplings from the SM prediction. In a general context, such a deviation can be parametrized as

$$
\begin{aligned}
V_{\text {self }} & =\frac{\lambda_{3}}{3 !} h^{3}+\frac{\lambda_{4}}{4 !} h^{4} \\
& \equiv \frac{1}{3 !} \lambda_{3, \mathrm{SM}}\left(1+\kappa_{3}\right) h^{3}+\frac{1}{4 !} \lambda_{4, \mathrm{SM}}\left(1+\kappa_{4}\right) h^{4},
\end{aligned}
$$

with $\kappa_{3}$ and $\kappa_{4}$ being free parameters. Pinning down the Higgs self-couplings with precision therefore is vital for probing the underlying physics and the nature of EWPT.

The measurements of the cubic Higgs coupling via various di-Higgs productions have been extensively studied so far. At hadron colliders, the main channels include gluon-fusion production, vector boson-fusion (VBF) production, top pair-associated production, and vector bosonassociated (VBA) production. At lepton colliders, the dominant channels are the $Z$ boson-associated production and the VBF production. The LHC has no sensitivity to the SM cubic Higgs coupling yet. But, the high-luminosity LHC, say, $\mathcal{L}=3 \mathrm{ab}^{-1} @ 14 \mathrm{TeV}$, is expected to be able to probe it with a precision of $\sim \mathcal{O}(1)$ in the gluon-fusion production [1,2], with an improvement of earlier analyses 
(see, e.g., Refs. [3,4]). At a future $100 \mathrm{TeV}$ hadron collider (for discussions at $27 \mathrm{TeV}$, see, e.g., Ref. [5]), the cubic Higgs coupling could be measured with a higher precision. For example, in the gluon-fusion channel, the cubic Higgs coupling could be measured with a precision of percent level $[6,7]$. The VBF production is found to be not quite sensitive $[8,9]$. The analysis for the top pair-associated production [10,11] and VBA productions [12] at a future hadron collider are still absent. As for the lepton colliders, the International Linear Collider (ILC) is able to measure the cubic Higgs coupling with a precision of $27 \%$ in the Zhh production at $500 \mathrm{GeV}$ with $\mathcal{L}=4 \mathrm{ab}^{-1}$ and a precision of $14 \%$ in the $\nu \nu h h$ production at $1 \mathrm{TeV}$ with $\mathcal{L}=2.5 \mathrm{ab}^{-1}$ [13]. The Compact Linear Collider (CLIC) is able to measure the cubic Higgs coupling with a precision of $54 \%$ in the $\nu \nu h h$ channel, with $\mathcal{L}=1.5 \mathrm{ab}^{-1}$ data at $1.4 \mathrm{TeV}$, and 29\%, with $\mathcal{L}=2 \mathrm{ab}^{-1}$ data at $3 \mathrm{TeV}$ [14].

To fully pin down the Higgs potential, we also need to measure the quartic Higgs coupling. The traditional wisdom for this is to measure the tri-Higgs productions. However, such measurements are known to be difficult, even at a future $100 \mathrm{TeV}$ hadron collider [15], due to the tiny cross section of tri-Higgs production and its weak dependence on the quartic Higgs coupling. The recent studies on the tri-Higgs productions in the most promising decay channel $b \bar{b} b \bar{b} \gamma \gamma$ showed that the sensitivity to probe $\kappa_{4}$ in the high-luminosity phase of the future hadron collider, say, $30 \mathrm{ab}^{-1} @ 100 \mathrm{TeV}$, is $\sim \mathcal{O}(10)$ [16,17] (for studies on the tri-Higgs searches in different decay channels, see Refs. $[18,19])$. This motivates the proposal in this article, say, to probe the quartic Higgs coupling via its loop corrections to the di-Higgs productions. We expect a combination of the di-Higgs and tri-Higgs measurements in the future to improve the precision of measuring the quartic Higgs coupling.

For the di-Higgs productions at colliders, there are two types of one-loop effects involving the quartic Higgs coupling. ${ }^{1}$ Both of them are independent of the choice of gauge fixing. The first type is the renormalization of the cubic Higgs coupling $\lambda_{3}{ }^{2}$, which is universal for different di-Higgs processes. The rest of the diagrams belong to the second type. They are irreducible and finite, yielding nontrivial corrections to the form factor of the relevant vertices such as $V V h h$. The two types of diagrams are reminiscent of the self-energy and the vertex corrections induced by the cubic Higgs coupling in the single Higgs production [20,21]. But, there exists a generic difference. The one-loop correction of the quartic Higgs coupling to the cubic Higgs coupling is logarithmically divergent. Its

\footnotetext{
${ }^{1}$ Unlike other di-Higgs productions, the gluon-fusion one does not involve the quartic coupling until the two-loop level. But we will not specify this subtlety below, upon the understanding.

${ }^{2}$ The quartic Higgs coupling also renormalizes the Higgs mass. But it can be fully resolved by the physical Higgs mass.
}

renormalization necessarily introduces a renormalizationscheme dependence on the interpretation of the experimental constraints for the cubic Higgs coupling.

A full treatment of these di-Higgs productions at oneloop level needs to embed the $\kappa$ scheme, essentially a parametrization of new physics corrections to the Higgs self-couplings, into an effective field theory (EFT) for the Higgs boson (for a review, see, e.g., Ref. [22]), and then take into account the loop effects from all relevant particles. Here, the EFT could be either the SM EFT, in which new particles get decoupled at a high-energy scale, or the Higgs Effective Field Theory (HEFT), which is known to describe the IR limit of some composite Higgs models (for a review, see, e.g., Ref. [23]), dilaton constructions [24,25], the SM extension with a nondecoupling heavy singlet scalar [26], etc. In these contexts, the quartic Higgs coupling is generally decoupled from other couplings relevant to the di-Higgs productions. In the HEFT, with its potential given by $V(h)=\sum_{n} a_{n}(h / v)^{n}$, this feature is generic. In the SM Effective Field Theory (SMEFT), the quartic Higgs coupling becomes decoupled as long as more than one higherdimensional operator is turned on. ${ }^{3}$ Interestingly, we observe that the one-loop diagrams with no quartic Higgs coupling involved (the summation of which is expected to be independent of gauge fixing and to involve the SM couplings and $\kappa_{3}$ only), though interfering with the tree-level $\kappa_{3}$ diagrams and the one-loop $\kappa_{4}$ diagrams, yield a next-to-leading-order (NLO) impact only for both the $\kappa_{3}$ and $\kappa_{4}$ sensitivity analysis at lepton colliders after a proper renormalization for $\lambda_{3}$. So, we will ignore such diagrams below. ${ }^{4}$ The QCD loop diagrams may yield nontrivial effects for the analysis at hadron colliders. In this paper, for a given di-Higgs process, we assume a universal QCD $K$ factor, which is independent of the corrections of the Higgs self-couplings.

The rest of the paper is organized as follows. In Sec. II, we will calculate the one-loop effects of the quartic Higgs coupling in renormalizing the cubic Higgs coupling and in correcting the $V V h h$ form factor. We will also discuss how to extract the $\kappa_{4}$ sensitivity in a way that is less dependent on the $\lambda_{3}$ renormalization scheme. The numerical calculations of the VBF and VBA di-Higgs productions at both lepton and hadron colliders are presented in Sec. III. We will analyze the sensitivities of the di-Higgs probe to the quartic Higgs coupling at the ILC and CLIC in Sec. IV. We will conclude in Sec. V.

\footnotetext{
${ }^{3}$ For discussions on the SMEFT phenomenology with $\mathcal{O}_{6}$ turned on, see, e.g., [27].

${ }^{4}$ Though a quinary Higgs coupling may appear in the BSM physics often, it has no contributions to the di-Higgs production at one-loop level, except renormalizing the cubic Higgs coupling. In that case, the effects of the quinary Higgs coupling can be fully absorbed by the counter-term.
} 

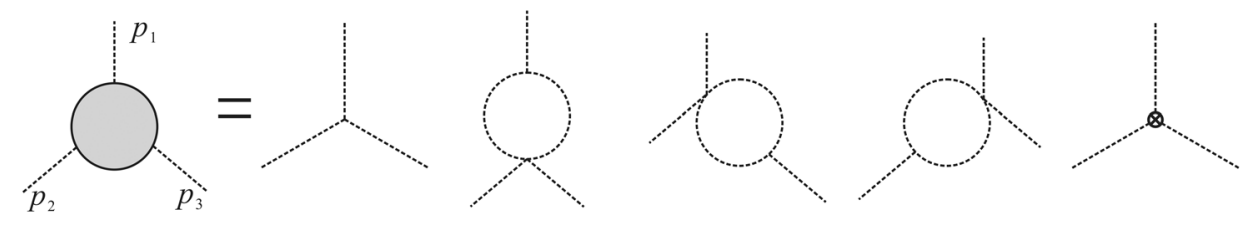

FIG. 1. One-loop Feynman diagrams for renormalizing the cubic Higgs coupling that are mediated by the quartic Higgs selfinteraction.

\section{ONE-LOOP EFFECTS OF THE QUARTIC HIGGS COUPLING}

The one-loop effects of the quartic Higgs coupling include (i) renormalizing the cubic Higgs coupling and (ii) modifying the form factor of the relevant vertices. The relevant Feynman diagrams are shown in Figs. 1-3, respectively. As discussed above, in general, we can assume that the quartic Higgs coupling is decoupled from other couplings involved in the di-Higgs productions, including the cubic Higgs coupling and the quartic couplings involving both Higgs and Goldstone bosons (for justification regarding this in the SMEFT, please see Appendix A). Then, its quantum corrections to the diHiggs productions are automatically guaranteed to be independent of the choice of gauge fixing. For the diagrams renormalizing the cubic Higgs coupling, no Goldstone bosons nor gauge bosons are involved where the gauge fixing is applied. These diagrams will contribute to the diHiggs productions in a universal way. For the diagrams modifying the $V V h h$ form factor, though both the gauge bosons and Goldstone bosons are involved, their summation yields a cancellation of the gauge dependence. These diagrams are finite and will contribute to the VBA and VBF di-Higgs productions. For the diagrams modifying the $t \bar{t} h h$, gt $\bar{t} h h$ (or Z $\bar{t} \bar{t} h h$ ), and ggt $\bar{t} h h$ form factors, again, no Goldstone bosons nor gauge bosons are involved. These diagrams are finite and will contribute to the gluon fusion and top quark-associated di-Higgs productions.

Computing the diagrams in Fig. 1 with the dimensional regularization, we obtain the tri-Higgs vertex

$$
i \Gamma\left(p_{1}^{2}, p_{2}^{2}, p_{3}^{2}\right)=i \lambda_{3}-i \frac{\lambda_{3} \lambda_{4}}{32 \pi^{2}} \sum_{j=1}^{3} \int_{0}^{1} d x\left(\frac{2}{\epsilon}-\gamma+\log (4 \pi)-\log \left[m_{h}^{2}-x(1-x) p_{j}^{2}\right]\right)+i \delta_{3}
$$

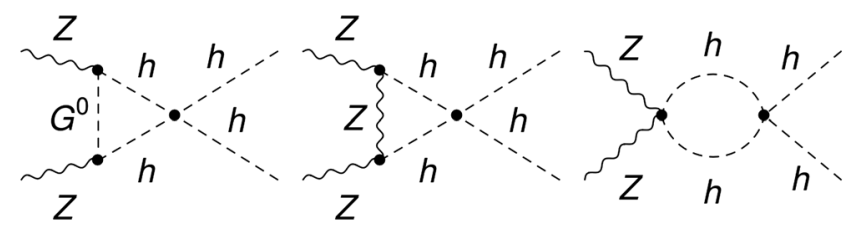

FIG. 2. One-loop Feynman diagrams for modifying the form factor of the quartic $V V h h$ vertex that are mediated by the quartic Higgs self-interaction. Here, $G$ is Goldstone boson, and we use the $\mathrm{Z}$ boson as an example.
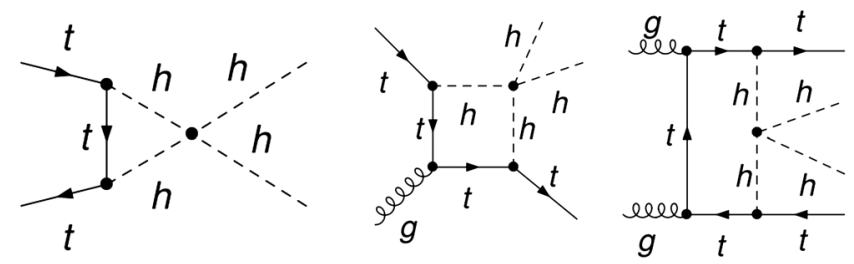

FIG. 3. One-loop Feynman diagrams for modifying the form factors of the $t \bar{t} h h, g t \bar{t} h h$, and ggt $\bar{t} h h$ vertices, which are mediated by the quartic Higgs self-interaction. where $\lambda_{3}$ is the renormalized cubic Higgs coupling and $\gamma=$ $0.577 \ldots$ is the Euler constant. We use $\delta_{3}$ to denote the counterterm schematically. This counterterm can arise from the higher-dimensional operators in the SMEFT (e.g., the dimension- 6 operator $\mathcal{O}_{6}$ ) or the $h^{3}$ term in the HEFT. Their coefficients then match onto the couplings between the Higgs field and the new fields in a UV-complete model which have been integrated out to define the EFT.

The renormalized cubic Higgs coupling $\lambda_{3}$ can be defined by properly choosing the $p_{j}^{2}$ values for $\Gamma\left(p_{1}^{2}, p_{2}^{2}, p_{3}^{2}\right)$. Since the three Higgs legs cannot be on shell at the same time, we will consider two schemes:

(i) Scheme 1.-Set $p_{j}^{2}=0$, and define $\lambda_{3} \equiv \Gamma(0,0,0)$. Equation (2.1) then becomes

$$
\begin{aligned}
& i \Gamma\left(p_{1}^{2}, p_{2}^{2}, p_{3}^{2}\right) \\
& \quad=i \lambda_{3}+i \frac{\lambda_{3} \lambda_{4}}{32 \pi^{2}} \sum_{j=1}^{3} \int_{0}^{1} d x \log \left[\frac{m_{h}^{2}-x(1-x) p_{j}^{2}}{m_{h}^{2}}\right] .
\end{aligned}
$$

This choice is effectively equivalent to the $\overline{\mathrm{MS}}$ renormalization scheme with $\mu=m_{h}$. 
(ii) Scheme 2.-Set $p_{1,2}^{2}=m_{h}^{2}, p_{3}^{2}=4 m_{h}^{2}$, and define $\lambda_{3} \equiv \Gamma\left(m_{h}^{2}, m_{h}^{2}, 4 m_{h}^{2}\right)$. In any di-Higgs productions, the cubic Higgs coupling always has two on-shell Higgs legs, and the third one is characterized by the di-Higgs invariant mass $p_{3}^{2}=M_{h h}^{2} \geq 4 m_{h}^{2}$. So, we define $\lambda_{3} \equiv \Gamma\left(m_{h}^{2}, m_{h}^{2}, 4 m_{h}^{2}\right)$, and Eq. (2.1) becomes

$$
\begin{aligned}
i \Gamma\left(p_{1}^{2}, p_{2}^{2}, p_{3}^{2}\right)=i \lambda_{3}+i \frac{\lambda_{3} \lambda_{4}}{32 \pi^{2}} \\
\quad \times\left(\sum_{j=1}^{3} \int_{0}^{1} d x \log \left[\frac{m_{h}^{2}-x(1-x) p_{j}^{2}}{m_{h}^{2}}\right]+2.37\right) .
\end{aligned}
$$

This choice is effectively equivalent to the $\overline{\mathrm{MS}}$ renormalization scheme with $\mu=0.67 m_{h}$.

The one-loop corrections of the quartic Higgs coupling to the $V V h h$ form factor is a summation of three terms in the $R_{\xi}$ gauge

$$
F[H H V V]=F_{1}+F_{2}+F_{3} .
$$

Here, $F_{i}$ denotes the contribution of the $i$ th diagram in Fig. 2 with the momentum of the incoming gauge bosons denoted as $k_{1}$ and $k_{2}$ :

$$
\begin{aligned}
& F_{1}=\left(\frac{i 2 m_{V}^{2}}{v}\right)^{2} \lambda_{4} \int \frac{d^{4} q}{(2 \pi)^{4}}\left[\frac{i}{q^{2}-m_{V}^{2}}\left(-g^{\mu \nu}+\frac{q^{\mu} q^{\nu}}{m_{V}^{2}}\right)-\frac{i}{q^{2}-\xi m_{V}^{2}} \frac{q^{\mu} q^{\nu}}{m_{V}^{2}}\right] \frac{i}{\left(q+k_{1}\right)^{2}-m_{h}^{2}} \frac{i}{\left(q+k_{2}\right)^{2}-m_{h}^{2}} \\
& F_{2}=\left(-\frac{m_{V}}{v}\right)^{2} \lambda_{4} \int \frac{d^{4} q}{(2 \pi)^{4}} \frac{i}{q^{2}-\xi m_{V}^{2}} \frac{i}{\left(q+k_{1}\right)^{2}-m_{h}^{2}} \frac{i\left(-2 q-k_{2}\right)^{\nu}\left(2 q+k_{1}\right)^{\mu}}{\left(q+k_{2}\right)^{2}-m_{h}^{2}} \\
& F_{3}=\frac{i 2 m_{V}^{2}}{v^{2}} \lambda_{4} \int \frac{d^{4} q}{(2 \pi)^{4}}\left[\frac{i}{q^{2}-m_{h}^{2}} \frac{i}{\left(q+k_{1}+k_{2}\right)^{2}-m_{h}^{2}}\right] .
\end{aligned}
$$

After a contraction with external massless fermion current or massive gauge bosons that are on shell, only the $q^{\mu} q^{\nu}$ term is left in $F_{2}$. Then, the summation of $F_{1}$ and $F_{2}$ leads to a cancellation of $\xi$ dependence, as we expected. One can also check that $F[H H V V]$ is UV finite, similar to the case of $F[H V V]$ discussed in Ref. [28].

The calculation of the one-loop corrections of the quartic Higgs coupling to the $t \bar{t} h h, g t \bar{t} h h$, and $g g t \bar{t} h h$ form factors is straightforward, based on the diagrams in Fig. 3. We do not show the results here, since below we will focus on the VBF and VBA di-Higgs productions.

With the renormalized cubic Higgs coupling $\lambda_{3}$ and the modified $V V h h$ form factor, we can parametrize the deviation of the cross section $\sigma$ from the SM prediction $\sigma_{0}$ in the relevant di-Higgs productions as

$\frac{\delta \sigma}{\sigma_{0}} \equiv \frac{\sigma-\sigma_{0}}{\sigma_{0}}=C_{31} \kappa_{3}+C_{32} \kappa_{3}^{2}+\kappa_{4}\left(C_{41}+C_{42} \kappa_{3}+C_{43} \kappa_{3}^{2}\right)$,

where $\kappa_{3}=\lambda_{3} v / 3 m_{h}^{2}-1$. The first two terms denote the contributions from the cubic Higgs coupling only, at the leading order that arises from the tree level. The rest arises from the interference between the $\kappa_{4}$ one-loop corrections and the tree-level amplitudes. We neglect the quadratic term in $\kappa_{4}$, given that it results from the interference between one-loop amplitudes. Then, the cubic and quartic Higgs couplings can be probed by measuring the di-Higgs production cross sections at colliders.

The interpretation of the collider sensitivities for probing $\kappa_{3}$ depends on the $\lambda_{3}$ renormalization scheme. But such a scheme dependence can be largely suppressed for $\kappa_{4}$, by marginalizing $\kappa_{3}$ in the $\chi^{2}$ analysis. This can be understood in the following way. Consider $N \geq 2$ observables $\left\{O_{i}\right\}$, which depend on two parameters $x$ and $y$ linearly:

$$
O_{i}=a_{i} x+b_{i} y
$$

The two parameters can be fit using the $\chi^{2}$ analysis, with

$$
\chi^{2}=\sum_{i=1}^{N}\left(\frac{O_{i}}{\sigma_{i}}\right)^{2}=\sum_{i=1}^{N}\left(\frac{a_{i} x+b_{i} y}{\sigma_{i}}\right)^{2} .
$$

Here, $\sigma_{i}$ is the measurement uncertainty of $O_{i}$. Then, the marginalized constraint for one of the two parameters, say, $y$, can be obtained by integrating $x$ out, given by

$\Delta \chi^{2}=\frac{\operatorname{det} M}{M_{x x}} \Delta y^{2}=\left[\frac{1}{2} \sum_{i, j=1}^{N} \frac{a_{i}^{2}}{\sigma_{i}^{2}} \frac{a_{j}^{2}}{\sigma_{j}^{2}}\left(\frac{b_{i}}{a_{i}}-\frac{b_{j}}{a_{j}}\right)^{2}\right]\left[\sum_{k=1}^{N} \frac{a_{k}^{2}}{\sigma_{k}^{2}}\right]^{-1} \Delta y^{2}$.

Here, $M$ is the inverse of the covariance matrix for $x$ and $y$. At $1 \sigma$ C.L., we have $\Delta \chi^{2}=1$, which yields 


$$
\Delta y=\sqrt{\left[\sum_{k=1}^{N} \frac{a_{k}^{2}}{\sigma_{k}^{2}}\right]\left[\frac{1}{2} \sum_{i, j=1}^{N} \frac{a_{i}^{2}}{\sigma_{i}^{2}} \frac{a_{j}^{2}}{\sigma_{j}^{2}}\left(\frac{b_{i}}{a_{i}}-\frac{b_{j}}{a_{j}}\right)^{2}\right]^{-1}} .
$$

To match with the discussions on the Higgs selfcouplings, we can make replacements: $(x, y) \rightarrow\left(\kappa_{3}, \kappa_{4}\right)$ and $\left(a_{i}, b_{i}\right) \rightarrow\left(C_{31}^{(i)}, C_{41}^{(i)}\right)$. At the leading order, $C_{31}^{(i)}$ is scheme independent, but $C_{41}^{(i)}$ is not. For any given pair of observables $O_{i}$ and $O_{j}$, we can eliminate $\kappa_{3}$, yielding the relation

$$
\frac{O_{i}}{C_{31}^{(i)}}-\frac{O_{j}}{C_{31}^{(j)}}=\left(\frac{C_{41}^{(i)}}{C_{31}^{(i)}}-\frac{C_{41}^{(j)}}{C_{31}^{(j)}}\right) \kappa_{4} \equiv \Delta C_{i j} \kappa_{4} .
$$

Since the left side of this equation is independent of the $\lambda_{3}$ renormalization scheme at the leading order, $\Delta C_{i j}$ should be nearly scheme independent, given that $\kappa_{4}$ by definition is a parameter independent of $\kappa_{3}$ or $\lambda_{3}$. Then, we are able to obtain $\Delta \kappa_{4}$ by applying Eq. (2.10), with the scheme dependence suppressed, if all pairs of $\left\{C_{41}^{(i)}, C_{41}^{(j)}\right\}$ are calculated with proper precisions. Note that the "if" condition is important for suppressing the linear-level scheme dependence. For example, if one were to combine the di-Higgs productions discussed above with the single Higgs productions in the analysis, the two-loop contributions of the quartic Higgs coupling to the latter channels would need to be incorporated. The nonlinear terms in Eq. (2.6), if turned on, may weaken this argument. But the scheme dependence introduced is of next-to-next-toleading order (NNLO) and could be further suppressed if the NNLO nonlinear terms, such as the ones proportional to $\kappa_{4}^{2}$, are properly calculated.

If there are two observables only, the formula for $\Delta \kappa_{4}$ is reduced to

$$
\Delta \kappa_{4}=\frac{\sqrt{\left(\sigma_{i} / C_{31}^{(i)}\right)^{2}+\left(\sigma_{j} / C_{31}^{(j)}\right)^{2}}}{\left|\Delta C_{i j}\right|} .
$$

Here, $\left|\sigma_{i} / C_{31}^{i}\right|$ and $\left|\sigma_{j} / C_{31}^{j}\right|$ represent the precision of measuring $\kappa_{3}$ via $O^{i}$ and $O^{j}$, respectively, with $\kappa_{4}$ being

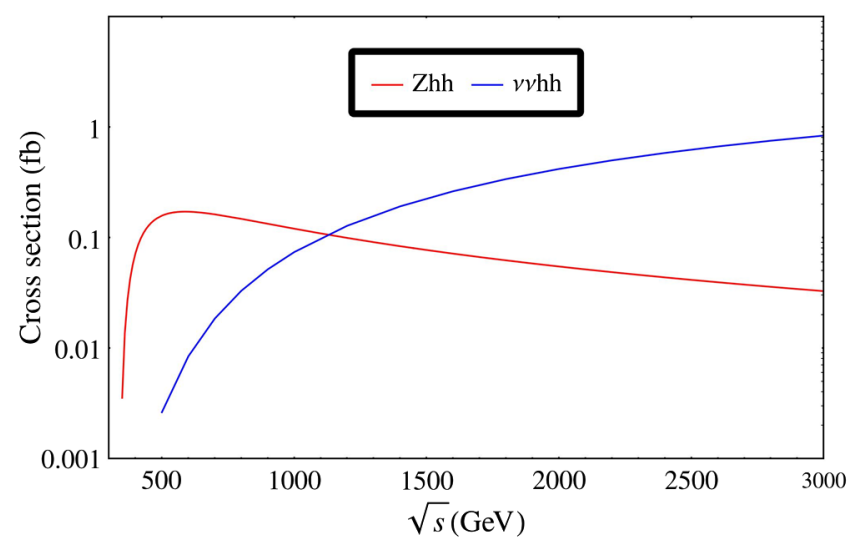

FIG. 4. The leading-order cross sections in the SM, as functions of the center-of-mass energy $\sqrt{s}$. The initial states are unpolarized.

turned off. An interesting observation is that a larger $\left|\Delta C_{i j}\right|$ tends to yield a higher precision for the $\kappa_{4}$ measurement. This can be the case when the two observables $O_{i}$ and $O_{j}$ constrain the $\kappa_{3}-\kappa_{4}$ plane in two clearly separated directions. Below, we will show how to optimize the measurement precision for $\kappa_{4}$ using this guideline.

\section{ANALYSES AT LEPTON AND HADRON COLLIDERS}

In this section, we calculate the one-loop contributions of the quartic Higgs coupling in the VBF and VBA di-Higgs productions at both lepton and hadron colliders. We use FEYNRULE [29] to generate the model file. The cross sections are then calculated with FEYNARTS3.8 and FORMCALC9.5 [30] using a factorization scale of $m_{h}=125 \mathrm{GeV}$, where the LoOPToOLs [31] is linked to calculate the loop integral. The electroweak input parameters in the analysis are chosen as $G_{F}=1.1663787 \times 10^{-5} \mathrm{GeV}^{-2}, m_{Z}=91.1876 \mathrm{GeV}$, and $m_{W}=80.385 \mathrm{GeV}$ [32]. For consistency checks, we compare the tree-level cross sections with those given by MAdGraPH@AMC2.3.3 [33] and CALCHEP3.6.27 [34]. Also, we have checked the values of the squared one-loop amplitudes at some given points in the phase space by comparing with the results calculated by hand.

TABLE I. The leading-order SM cross sections and the parametrization of the $\kappa_{3}, \kappa_{4}$ contributions for the Zhh and $\nu \nu h h$ di-Higgs

\begin{tabular}{|c|c|c|c|c|c|c|c|}
\hline Chann & & $\sigma_{0}(\mathrm{fb})$ & $C_{31}$ & $C_{32}$ & $C_{41}$ & $C_{42}$ & $C_{43}$ \\
\hline \multirow[t]{3}{*}{ ILC } & Zhh $(500 \mathrm{GeV})$ & 0.232 & 0.564 & 0.0965 & -0.00517 & -0.00390 & -0.000810 \\
\hline & Zhh $(1 \mathrm{TeV})$ & 0.166 & 0.350 & 0.0913 & -0.00271 & -0.00181 & -0.000541 \\
\hline & $\nu \nu h h(1 \mathrm{TeV})$ & 0.159 & -1.20 & 1.10 & -0.00327 & 0.00790 & -0.00750 \\
\hline \multirow[t]{3}{*}{ CLIC } & Zhh $(1.4 \mathrm{TeV})$ & 0.0833 & 0.263 & 0.0827 & -0.00186 & -0.00122 & -0.000422 \\
\hline & $\nu \nu h h(1.4 \mathrm{TeV})$ & 0.191 & -0.965 & 0.819 & -0.0024 & 0.00541 & -0.00505 \\
\hline & $\nu \nu h h(3 \mathrm{TeV})$ & 0.825 & -0.645 & 0.488 & -0.00119 & 0.00251 & -0.00247 \\
\hline
\end{tabular}
productions at lepton colliders. Here, the ILC beam is polarized as $P\left(e^{-}, e^{+}\right)=(-0.8,0.3)$ at $500 \mathrm{GeV}$ and $P\left(e^{-}, e^{+}\right)=(-0.8,0.2)$ at $1 \mathrm{TeV}$. 


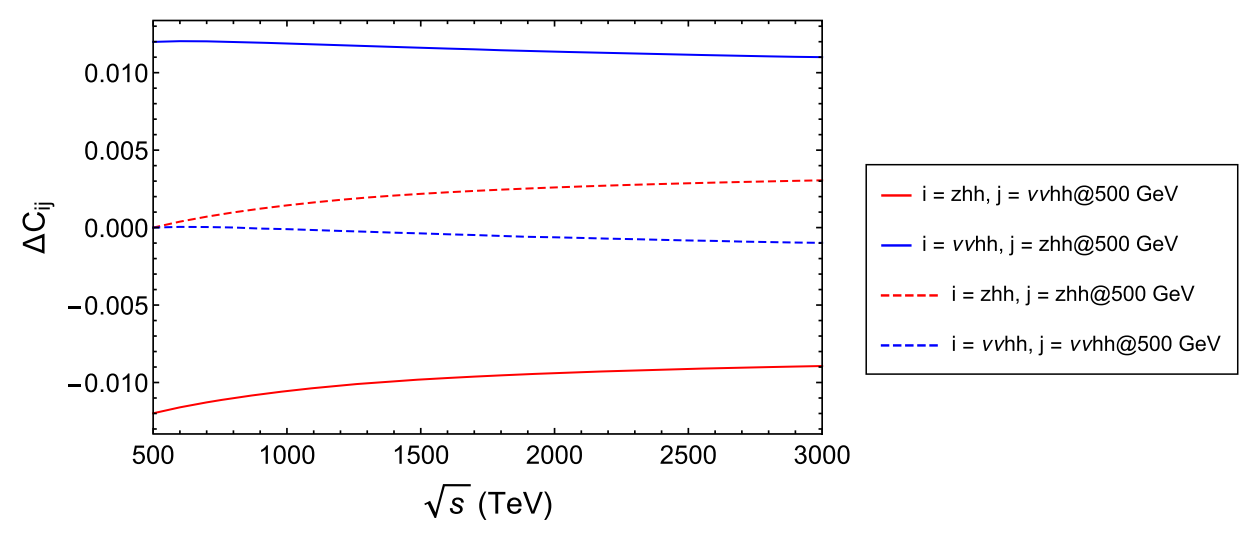

FIG. 5. $\Delta C_{i j}$ for the observable pairs $\left\{O_{i}, O_{j}\right\}$ available in the $e^{+} e^{-} \rightarrow Z h h$ and $e^{+} e^{-} \rightarrow \nu \nu h h$ channels. Here, $O_{j}$ represents the reference observable, with $\sqrt{s}$ varied for $O_{i}$ from $500 \mathrm{GeV}$ to $3 \mathrm{TeV}$.

\section{A. Lepton colliders}

At lepton colliders, the main di-Higgs production processes include the $Z$-associated production $e^{+} e^{-} \rightarrow Z h h$ and the VBF production $e^{+} e^{-} \rightarrow \nu \nu h h$. Though they could be kinematically turned on, the VBF production $e^{+} e^{-} \rightarrow$ $e^{+} e^{-} h h$ and the top pair-associated production $e^{+} e^{-} \rightarrow$ $t \bar{t} h h$ suffer a suppression of cross section. So, we will focus on the former two channels. Figure 4 shows their leadingorder cross sections in the SM, as functions of the center-ofmass energy $\sqrt{s}$, with an unpolarized initial state. The cross section for the Zhh process reaches the peak at $\sqrt{s} \sim$ $500 \mathrm{GeV}$ and then slowly decreases due to an $s$-channel suppression. As for the VBF production of $\nu \nu h h$, due to the $t$-channel contributions mediated by the $W$ boson, its cross section keeps growing up to a few TeV. In Table I, we show the leading-order SM cross sections and the coefficients defined in Eq. (2.6) for these two processes, in different collider configurations. The cubic Higgs coupling is renormalized in scheme 1 . Note that the beam polarization does not modify the values of $C_{3 a}$ and $C_{4 b}$ but changes the total cross section only.

As we demonstrated in Sec. II, the $\Delta C_{i j}$ defined in Eq. (2.11) is independent of the $\lambda_{3}$ renormalization scheme at the linear level. Particularly, a larger $\left|\Delta C_{i j}\right|$ tends to yield a higher precision for the $\kappa_{4}$ measurement, after $\kappa_{3}$ is marginalized. For optimizing the collider sensitivities and potentially its configuration design, therefore, it is helpful to have the information on $\left|\Delta C_{i j}\right|$ for various observable pairs available. In Fig. 5, we show $\Delta C_{i j}$ for the observables available in the $Z h h$ and $\nu \nu h h$ channels. The dashed and solid lines denote the cases in which the two observables are from the same and different channels, respectively. The red and blue colors represent different choices for the reference observable $O_{j}$. Then, we show the $\sqrt{s}$ dependence of $\Delta C_{i j}$ by varying $\sqrt{s}$ from $500 \mathrm{GeV}$ to $3 \mathrm{TeV}$ for $O_{i}$. Interestingly, the two observables, if arising from the $\mathrm{Zhh}$ and $\nu$ hh channels separately, result in a $\left|\Delta C_{i j}\right|$ of $\mathcal{O}\left(10^{-2}\right)$. This is several times or even one order larger than that obtained in the complementary cases and is not sensitive to the value of $\sqrt{s}$. Indeed, such a pair of observables has clearly separated degenerate directions at the $\kappa_{3}-\kappa_{4}$ plane. A combination of them will be very important for optimizing the sensitivities to probe $\kappa_{4}$.

\section{B. Hadron colliders}

The main di-Higgs production processes at hadron colliders include the gluon-fusion production $(g g \rightarrow h h)$, the top pair-associated production $(p p \rightarrow \bar{t} t h h)$, the VBF production $(p p \rightarrow h h j j)$, and the VBA production $(p p \rightarrow V h h, V=Z, W)$. For all of these processes, the cross sections increase as $\sqrt{s}$ increases from 14 to $100 \mathrm{TeV}$. At $100 \mathrm{TeV}$, the gluon-fusion cross section is around $1 \mathrm{pb}$;

TABLE II. The leading-order SM cross sections and the parametrization of the $\kappa_{3}, \kappa_{4}$ contributions for the $Z h h / W h h$ and $j j h h$ diHiggs productions at hadron colliders. For simplicity, we only include the contributions arising from the (anti)up and (anti)down quarks initiated processes. Also, we require the $W$ boson to be electrically positive in the Whh production.

\begin{tabular}{lccccccc}
\hline \hline Channels & & $\sigma_{0}(\mathrm{fb})$ & $C_{31}$ & $C_{32}$ & $C_{41}$ & $C_{42}$ & $C_{43}$ \\
\hline \multirow{2}{*}{$14 \mathrm{TeV}$} & $j j h h$ & 1.26 & -0.781 & 0.688 & -0.00233 & -0.00466 & -0.00426 \\
& $Z h h$ & 0.274 & 0.496 & 0.0954 & -0.00441 & -0.00327 & -0.000738 \\
& $W h h$ & 0.268 & 0.521 & 0.109 & -0.0041 & -0.00331 & -0.000807 \\
$100 \mathrm{TeV}$ & $j j h h$ & 59.3 & -0.537 & 0.411 & -0.00123 & 0.00238 & -0.00220 \\
& $Z h h$ & 2.95 & 0.454 & 0.091 & -0.00416 & -0.00293 & -0.000677 \\
& $W h h$ & 2.49 & 0.483 & 0.105 & -0.00386 & -0.003 & -0.00075 \\
\hline \hline
\end{tabular}




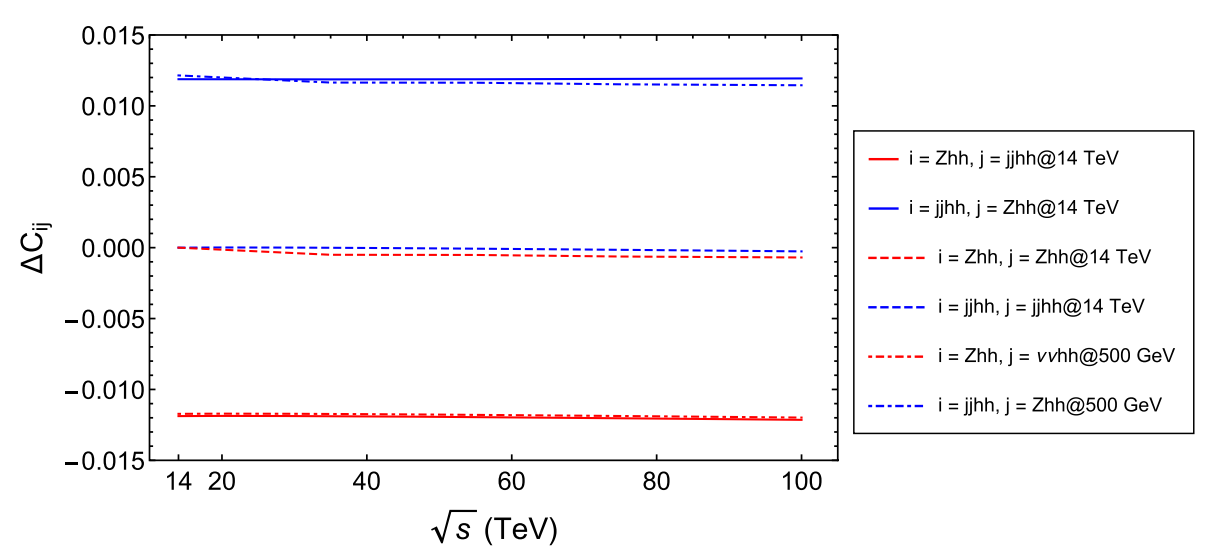

FIG. 6. $\Delta C_{i j}$ for the observable pairs $\left\{O_{i}, O_{j}\right\}$ available in the $p p \rightarrow Z h h$ and $p p \rightarrow j j h h$ channels. Here, $O_{j}$ represents the reference observable, with $\sqrt{s}$ varied for $O_{i}$ from 14 to $100 \mathrm{TeV}$.

the $h h j j$ and $t$ th $h$ ones are roughly 80-90 fb; the $V h h$ ones are several femtobarns [35,36]. For illustration purposes, we will focus on the VBA and VBF productions.

Table II shows the leading-order cross sections in the SM and the coefficients defined in Eq. (2.6) for the VBA and VBF productions, at 14 and $100 \mathrm{TeV}$. Here, we find the contribution from the VBF productions at hadron colliders by imposing a set of universal VBF selection cuts as [9]

$p_{T, j}>25 \mathrm{GeV}, \quad \Delta R_{j j}>4, \quad M_{j j}>600 \mathrm{GeV}$

except a rapidity cut $\left|\eta_{j}\right|<4.5$ at $14 \mathrm{TeV}$ and $\left|\eta_{j}\right|<10$ at $100 \mathrm{TeV}$. The cubic Higgs coupling is renormalized in scheme 1.

Similar to the analyses at lepton colliders, the knowledge on $\left|\Delta C_{i j}\right|$ is helpful for optimizing the sensitivities at the hadron collider to probe the quartic Higgs coupling. In Fig. 6, we show $\Delta C_{i j}$ for the observable pairs, which are available in the $Z h h$ and $j j h h$ channels. ${ }^{5}$ We use the red and blue colors to denote the $Z h h$ and the $j j h h$ as $O_{i}$, respectively. The lines of different styles (solid, dashed, and dotted-dashed) represent different reference observables $O_{j}$ for a given $O_{i}$. Then, we show the $\sqrt{s}$ dependence of $\Delta C_{i j}$ by varying $\sqrt{s}$ from 14 to $100 \mathrm{TeV}$ for $O_{i}$. The two observables, if arising from the $Z h h / W h h$ and the $j j h h$ at the hadron collider separately, result in a $\left|\Delta C_{i j}\right|$ of $\mathcal{O}\left(10^{-2}\right)$. This magnitude is several times or even one order larger than that obtained in the cases in which both observables are from the $Z h h / W h h$ channels or both are from the $j j h h$ channel and is not very sensitive to the value of $\sqrt{s}$. These observations are similar to what we had at lepton colliders. So, a combination of such a pair of observables is very important for optimizing the

\footnotetext{
${ }^{5}$ The $Z h h$ and $W h h$ productions share similar dependence on $\kappa_{3}$ and $\kappa_{4}$, as is indicated in Table II. Considering this, we do not show the Whh-related curves in Fig. 6.
}

sensitivities to probe $\kappa_{4}$ at hadron colliders. This conclusion can be generalized to the combination of two observables that are defined at lepton colliders and hadron colliders, separately. As is shown in Fig. 6, the jjhh and the $Z h h$ at hadron colliders can result in a $\left|\Delta C_{i j}\right|$ of $\mathcal{O}\left(10^{-2}\right)$ as well, by pairing with the $Z h h$ and the $\nu \nu h h$ at lepton colliders, respectively.

\section{COLLIDER SENSITIVITIES TO THE HIGGS SELF-COUPLINGS}

In this section, we reinterpret the projected precisions of the di-Higgs measurements as the sensitivities to probe both the cubic and quartic Higgs couplings. At hadron colliders, the sensitivity of measuring the VBA production at the LHC is poor [12], and the study on a future collider is absent. The VBF production, on the other hand, is not quite sensitive to the cubic Higgs coupling [8,9], and new analysis strategies have yet to be developed. So, we will focus on the analysis at lepton colliders. For simplification, we assume that the Higgs self-couplings only yield negligible modifications for the signal efficiency of the SM contributions. Then, the projected precisions as summarized in Table III can be directly applied to our analysis below, using the parametrization in Eq. (2.6). For the convenience of discussions, we define two ILC scenarios:

(i) $\mathrm{ILC} 1=\mathrm{ILC} \quad\left(500 \mathrm{GeV}, 4 \mathrm{ab}^{-1}+1 \mathrm{TeV}, 2.5 \mathrm{ab}^{-1}\right.$ [13]);

(ii) $\operatorname{ILC} 2=\operatorname{ILC}\left(500 \mathrm{GeV}, 4 \mathrm{ab}^{-1}+1 \mathrm{TeV}, 8 \mathrm{ab}^{-1}\right.$ [37]).

Figure 7 shows the sensitivity contours of measuring $\kappa_{3}$ and $\kappa_{4}$ at $1 \sigma$ C.L., at the ILC and CLIC. Here, the cubic Higgs coupling is renormalized in scheme 1. In this figure, the yellow region is defined by the perturbative unitarity bound of the $h h \rightarrow h h$ scattering (the derivation is presented in Appendix B). This unitarity requirement sets a range between $\sim \pm 65$ for $\kappa_{4}$, within which $\kappa_{3}$ is allowed to vary from $\sim-9$ to $\sim 7$. The brown and blue circles represent the sensitivities of the ILC1 and the ILC2, respectively. 
TABLE III. Projected precision of the di-Higgs measurements at $1 \sigma$ C.L., at the ILC and CLIC. The numbers in red are obtained by naively rescaling the signal rates.

\begin{tabular}{|c|c|c|c|c|c|}
\hline \multirow{2}{*}{$\begin{array}{l}\delta \sigma / \sigma_{\mathrm{SM}} \\
\text { Operating } \\
\text { scenarios }\end{array}$} & \multicolumn{3}{|c|}{ ILC } & \multicolumn{2}{|c|}{ CLIC } \\
\hline & $500 \mathrm{GeV}, 4 \mathrm{ab}^{-1}$ & $1 \mathrm{TeV}, 2.5 \mathrm{ab}^{-1}$ & $1 \mathrm{TeV}, 8 \mathrm{ab}^{-1}$ & $1.4 \mathrm{TeV}, 1.5 \mathrm{ab}^{-1}$ & $3 \mathrm{TeV}, 3 \mathrm{ab}^{-1}[38$ \\
\hline Zhh & $15 \%$ [13] & $22.5 \%[13]$ & $12.6 \%[13]$ & $30 \%$ [13] & \\
\hline$\nu \nu h h$ & $\ldots$ & $16.8 \%[13]$ & $9.4 \%[13]$ & $44 \%[14]$ & $16.3 \%[14]$ \\
\hline
\end{tabular}

In both scenarios, the ILC yields an exclusion limit for $\kappa_{3}$ and $\kappa_{4}$ well within the perturbative regime. ${ }^{6}$ This can be understood, since the ILC sensitivities benefit a lot from:

(i) the combination of the Zhh and $\nu \nu h h$ observables, which are characterized by relatively large $\left|\Delta C_{i j}\right|$ values of $\mathcal{O}\left(10^{-2}\right)$

(ii) the good precisions for measuring the $Z h h$ at $500 \mathrm{GeV}$ (almost maximized cross section, high luminosity) and the $\nu \nu h h$ at $1 \mathrm{TeV}$ (large cross section, high luminosity).

The purple circle represents the CLIC sensitivities by combining the measurements of $\mathrm{Zhh}$ at $1.4 \mathrm{GeV}$ and $\nu \nu h h$ at 1.4 and $3 \mathrm{TeV}$. As a comparison, it is difficult for the CLIC to reach an exclusion limit for $\kappa_{4}$ within the perturbative regime. Its sensitivities suffer from both the suppressed $Z h h$ cross section at a higher beam energy scale and the relatively low luminosity.

Given its potential in probing the Higgs self-interactions, let us look into the ILC analysis and sensitivities in more detail. In Fig. 8, we present the ILC sensitivities in both scheme 1 and scheme 2 of the $\lambda_{3}$ renormalization, with and without the nonlinear terms in Eq. (2.6). At the linear level, the exclusion contours at the $\kappa_{3}-\kappa_{4}$ plane are an ellipse with the major axis being close to the $\kappa_{4}$ direction. As is indicated in the left panel, the change from scheme 1 to scheme 2 yields a counterclockwise rotation for the exclusion contours. The nonlinear terms deform these ellipses. Compared to scheme 2, the ellipse orientation in scheme 1 restricts $\kappa_{3}$ to be smaller and makes the nonlinear effects less important. In the right panel, the sensitivities to probe $\kappa_{3}$ and $\kappa_{4}$ are shown by marginalizing $\kappa_{4}$ and $\kappa_{3}$, respectively, in the $\chi^{2}$ fit. The $\kappa_{3}$ sensitivity depends strongly on the $\lambda_{3}$ renormalization scheme by definition, while the $\kappa_{4}$ sensitivity is nearly scheme independent at the linear level, as we advertised in Sec. II. The scheme dependence is mainly introduced via the nonlinear terms in Eq. (2.6) in this context. The allowed ranges for $\kappa_{4}$ can then vary by a few percent between scheme 1 and scheme 2 .

\footnotetext{
${ }^{6} \mathrm{By}$ marginalizing $\kappa_{3}$, the circled ILC1 and ILC2 regions with $\kappa_{3} \sim 1-2$ and $\kappa_{4}>60$ yield a $\kappa_{4}$ falling outside the unitarity bound. Also, these regions could be excluded by combining with the single Higgs productions, e.g., the $Z h$ and $\nu \nu h$ ones, at future lepton colliders [39]. So, we will not consider it here.
}

To illustrate the scheme dependence more clearly, we present in Fig. 9 the $\kappa_{4}$ sensitivity in the ILC2 scenario for a wide range of the renormalization scale $\mu$, say, from 50 to $500 \mathrm{GeV}$. The difference between the yellow and the light blue regions shows the scheme dependence introduced via nonlinear terms in Eq. (2.6). Scheme 1 turns out to yield the almost minimal discrepancy between the linear and nonlinear results. For a smaller or larger renormalization scale, the constrained region shifts downward, yielding a less positive upper limit and a more negative lower limit. In a scenario with lower sensitivities, say ILC1, the nonlinear effects would be more significant.

In all, the ILC has a potential to probe $\left|\kappa_{4}\right|$ as small as $\sim 25$ in the ILC1 scenario and $\sim 20$ in the ILC2 scenario, respectively, at $1 \sigma$ C.L., in a $\lambda_{3}$ renormalization scheme in which nonlinear effects are minimized. Such a sensitivity is comparable to the one that could be achieved by measuring the tri-Higgs production at a high-luminosity future hadron collider, say, $30 \mathrm{ab}^{-1} @ 100 \mathrm{TeV}[16,17]$.

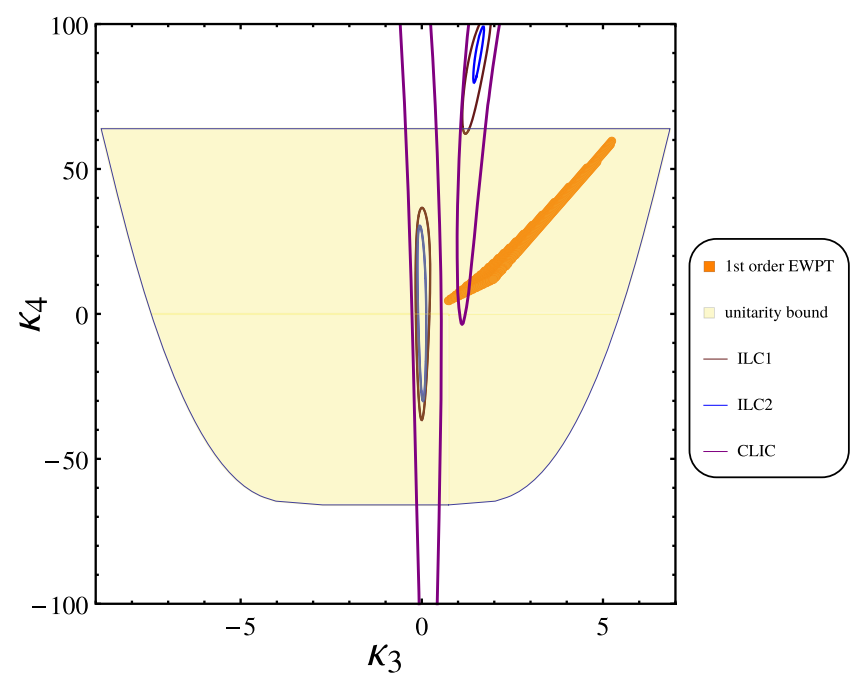

FIG. 7. The sensitivity contours of measuring $\kappa_{3}$ and $\kappa_{4}$ at $1 \sigma$ C.L., at the ILC and CLIC. The yellow region is perturbatively unitarity safe. As a benchmark, we indicate the region that is favored by first-order EWPT in the SMEFT with the $\mathcal{O}_{6}$ and $\mathcal{O}_{8}$ operators turned on (the discussions are presented in Appendix C) in orange. 

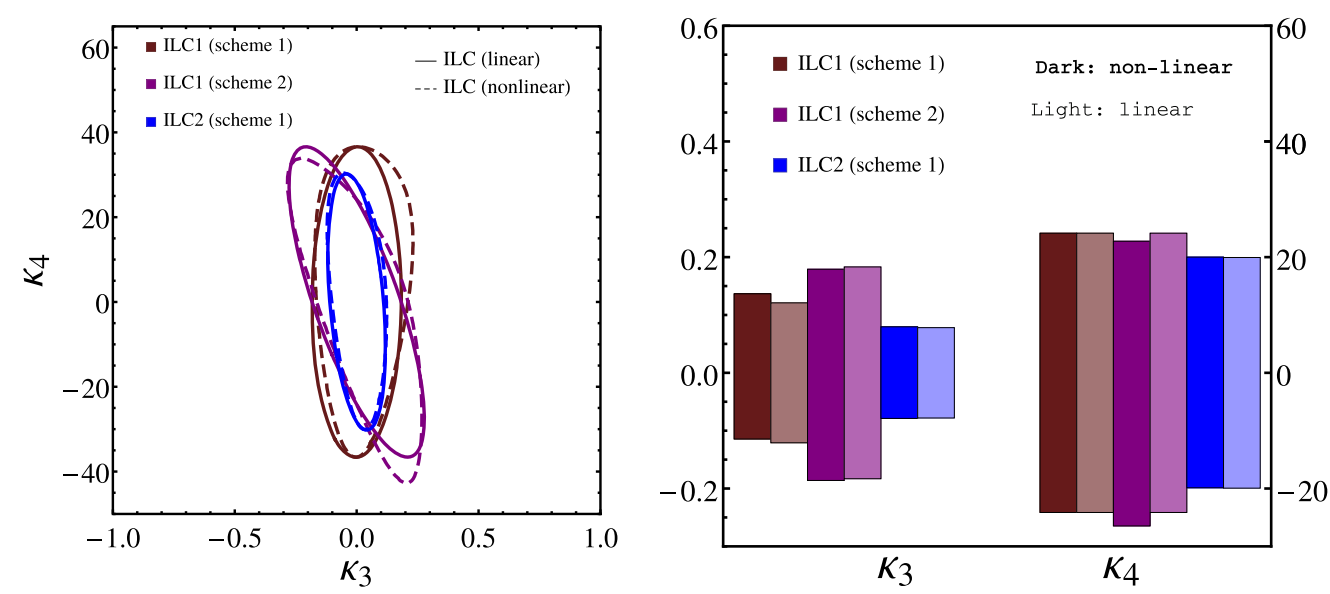

FIG. 8. The ILC sensitivities of measuring $\kappa_{3}$ and $\kappa_{4}$ at $1 \sigma$ C.L., in different $\lambda_{3}$ renormalization schemes, with and without the nonlinear terms in Eq. (2.6). In the right panel, the sensitivities to probe $\kappa_{3}$ and $\kappa_{4}$ are presented with $\kappa_{4}$ and $\kappa_{3}$ marginalized, respectively.

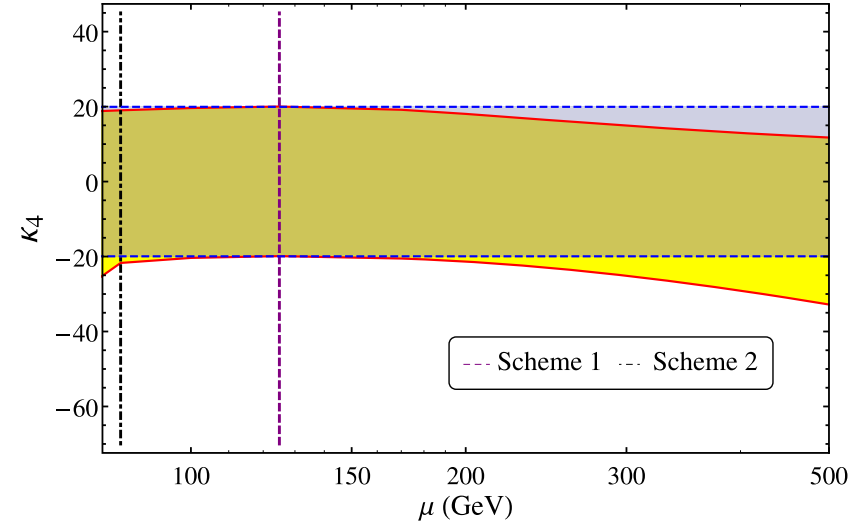

FIG. 9. The renormalization scheme dependence of the sensitivities of measuring $\kappa_{4}$ in the ILC2 scenario. The purple dashed and black dotted-dashed vertical lines correspond to scheme 1 and scheme 2, respectively. The shaded light blue region represents the sensitivity at the linear level, and the yellow region includes the nonlinear terms in Eq. (2.6) in addition.

\section{CONCLUSION}

The Higgs self-interactions play a crucial role for exploring the underlying mechanisms of electroweak symmetry breaking and the nature of the phase transition involved. Motivated by this, we proposed to probe the quartic Higgs self-interaction at lepton and hadron colliders, via the di-Higgs productions. We analyzed the corrections of the quartic Higgs coupling to the VBF and VBA di-Higgs productions at the one-loop level. Such an effect is independent of the gauge fixing, if the quartic Higgs coupling is decoupled from other couplings in the given context. In the calculations, we ignored the one-loop diagrams with no quartic Higgs coupling involved. These diagrams yield a NLO impact only for the sensitivity analysis of $\kappa_{3}$ and $\kappa_{4}$ at lepton colliders, after a proper renormalization for $\lambda_{3}$. One notable observation in the analysis is that the observables from the VBF and VBA
di-Higgs productions probe the $\kappa_{3}-\kappa_{4}$ plane in two clearly separated directions, at both lepton and hadron colliders. A combination of these two channels therefore is important for optimizing the collider sensitivities. With this guideline, we analyzed the ILC and CLIC sensitivities. We are able to extract the sensitivity on $\kappa_{4}$, which is nearly independent of the $\lambda_{3}$ renormalization scheme at the linear level, by marginalizing the cubic Higgs coupling in the $\chi^{2}$ analysis. Then, in a $\lambda_{3}$ renormalization scheme in which the nonlinear effects are almost minimized, we found that the ILC has the potential to measure the quartic Higgs coupling, normalized by its SM value, with a marginalized precision of $\sim \pm 25$ in the ILC1 scenario and $\sim \pm 20$ in the ILC2 scenario at $1 \sigma$ C.L.

The collider sensitivities could be further improved by utilizing the di-Higgs invariant mass distribution of the diHiggs events. In the analysis pursued, we have assumed that new physics does not significantly modify the kinematics of the SM di-Higgs events. To look into this further, we show the SM cross sections and the values of $C_{3 a}$ and $C_{4 b}$ in the di-Higgs invariant mass bins of $e^{-} e^{+} \rightarrow Z h h$ and $e^{-} e^{+} \rightarrow \nu \nu h h$ at ILC, in Tables IV and V of Appendix D, respectively. It is easy to see, though the $\frac{C_{41}}{C_{31}}$ defined in the Zhh channel is not very sensitive to the $m_{h h}$ values, a relatively small $m_{h h}$ value yields a more negative $\frac{C_{41}}{C_{31}}$ in the $\nu \nu h h$ channel and hence a larger $\left|\Delta C_{i j}\right|$ between the two channels. Additionally, both channels become more sensitive to $\kappa_{3}$ in the low $m_{h h}$ region, with a larger $\left|C_{31}\right|$ value. According to Eq. (2.11), therefore, the collider sensitivities could be further improved by requiring relatively small $m_{h h}$ for the di-Higgs events. Furthermore, if Eq. (2.12) is applied to the pair of observables $Z h h$ at $500 \mathrm{GeV}$ and $\nu \nu h h$ at $1 \mathrm{TeV}$, we can check

$$
\left(\frac{\sigma_{Z h h}}{C_{31}^{Z h h}}\right)^{2} \gg\left(\frac{\sigma_{\nu \nu h h}}{C_{31}^{\nu \nu h h}}\right)^{2}
$$


Thus, by improving the measurement precision for the $Z h h$ at $500 \mathrm{GeV}$, if sizably, the sensitivities for probing $\kappa_{4}$ could be significantly improved.

We need to keep in mind that the di-Higgs productions could be contaminated by some other new physics, via, e.g., the wave function renormalization of gauge bosons or the Higgs boson, the definition shift of the electroweak parameters, or the introduction of new vertices. Here, we have turned off all of these effects and simply assumed that they can be constrained sufficiently well for our purpose, by the electroweak and Higgs precision measurements at future colliders (for recent studies, see, e.g., Refs. [40-43]).

Given the significance of measuring the Higgs selfinteractions in particle physics, it is worthwhile to pursue a more systematic and complete analysis on its collider sensitivities. We can extend the analysis from lepton colliders to hadron colliders, particularly to the next-generation hadron colliders. More di-Higgs production channels can be taken into account, such as the gluon-fusion and top quark-associated processes, in that case. The leading-order effects of the quartic Higgs coupling appear at two- and oneloop level, respectively. We may also incorporate the triHiggs productions at both lepton colliders and hadron colliders in the analysis. The observables arising from these channels could be characterized by a $\left|\Delta C_{i j}\right|$ of $\mathcal{O}\left(10^{-2}\right)$ as well and further improve the marginalized precision of $\kappa_{4}$. Additionally, the quartic Higgs coupling contributes to the single Higgs productions (e.g., $Z h$ and $\nu \nu h$ ) at two-loop level, which in turn may facilitate the probe for the quartic Higgs coupling. To end the discussion, we would stress again that to probe $\kappa_{4}$ by combining the di-Higgs productions and other Higgs channels the $C_{41} / C_{31}$ for both need to be calculated with proper precisions, to suppress the scheme dependence of the $\lambda_{3}$ renormalization at least at the linear level. We leave a full study on these to future work.

\section{ACKNOWLEDGMENTS}

We would like to thank Tao Han, Sunghoon Jung, Zhen Liu, and Lian-Tao Wang for useful discussions. K.-F. L. would thank Xiaozhou Li, Yu Zhang, and Shaoming Wang for discussions on the tool of loop integration. T. L. is supported in part by the General Research Fund (GRF) under Grant No. 16312716 and in part by the GRF under Grant No. 16302117, both of which are issued by the Research Grants Council of Hong Kong SAR. J.R. is supported in part by the Natural Sciences and Engineering Research Council of Canada.

Note added.-Recently, Ref. [44] appeared, and it partially overlaps with this one in analyzing the one-loop corrections of the quartic Higgs coupling to the Zhh and $\nu \nu h h$ productions at lepton colliders. But our work is different from Ref. [44] in the following aspects: (i) We developed a general guideline for optimizing the collider sensitivities of probing the quartic Higgs coupling, based on Eq. (2.11). (ii) We analyzed the one-loop corrections of the quartic Higgs coupling to the $Z h h / W h h$ and $j j h h$ productions at hadron colliders as well. (III) We presented the ILC sensitivities for probing $\kappa_{4}$ by marginalizing $\kappa_{3}$ in the $\chi^{2}$ analysis and discuss the scheme dependence of the $\lambda_{3}$ renormalization in detail.

\section{APPENDIX A: GENERAL HIGGS POTENTIAL AND COUPLINGS IN SMEFT}

In this Appendix, we find the relation between Higgs self-couplings and Higgs-Goldstone couplings in SMEFT. A general Higgs potential here can be parametrized as a general function,

$V=F\left(H^{\dagger} H\right), \quad H^{\dagger} H=\frac{1}{2}(h+v)^{2}+\frac{1}{2} \pi^{0} \pi^{0}+\pi^{+} \pi^{-}$.

Given the electroweak scale $v$ and the Higgs mass $m_{h}$, the first two derivatives of the general function are determined by $\partial V /\left.\partial h\right|_{v}=0$ and $\partial^{2} V /\left.\partial h^{2}\right|_{v}=m_{h}^{2}$,

$$
F_{v}^{\prime}=0, \quad F_{v}^{\prime \prime}=m_{h}^{2} / v^{2},
$$

where the subscript $v$ denotes the quantity evaluated at $h=v, \pi^{i}=0$. After substituting (A2), the cubic couplings of Higgs and Goldstone bosons turn out to depend on the general function only up to the second derivative,

$$
\begin{gathered}
\Gamma\left(\pi^{0} \pi^{0} h\right)=\left.\frac{2 \partial}{\partial\left(\pi^{0} \pi^{0}\right)} \frac{\partial V}{\partial h}\right|_{v}=v F_{v}^{\prime \prime}=\frac{m_{h}^{2}}{v}, \\
\Gamma\left(\pi^{+} \pi^{-} h\right)=\left.\frac{\partial}{\partial\left(\pi^{+} \pi^{-}\right)} \frac{\partial V}{\partial h}\right|_{v}=v F_{v}^{\prime \prime}=\frac{m_{h}^{2}}{v} .
\end{gathered}
$$

So, for an arbitrary Higgs potential in (A1), they remain the same as in the SM.

The cubic Higgs coupling and the quartic HiggsGoldstone-boson quartic couplings further depend on the third derivative of the general function,

$$
\begin{gathered}
\Gamma(h h h)=\left.\frac{\partial^{3} V}{\partial h^{3}}\right|_{v}=3 v F_{v}^{\prime \prime}+v^{3} F_{v}^{\prime \prime \prime}=\frac{3 m_{h}^{2}}{v}\left(1+\kappa_{3}\right) \\
\Gamma\left(\pi^{0} \pi^{0} h h\right)=\left.\frac{2 \partial}{\partial\left(\pi^{0} \pi^{0}\right)} \frac{\partial^{2} V}{\partial h^{2}}\right|_{v}=F_{v}^{\prime \prime}+v^{2} F_{v}^{\prime \prime \prime}=\frac{m_{h}^{2}}{v^{2}}\left(1+3 \kappa_{3}\right) \\
\Gamma\left(\pi^{+} \pi^{-} h h\right)=\left.\frac{\partial}{\partial\left(\pi^{+} \pi^{-}\right)} \frac{\partial^{2} V}{\partial h^{2}}\right|_{v}=F_{v}^{\prime \prime}+v^{2} F_{v}^{\prime \prime \prime}=\frac{m_{h}^{2}}{v^{2}}\left(1+3 \kappa_{3}\right),
\end{gathered}
$$

where $\kappa_{3} \equiv v^{2} F_{v}^{\prime \prime \prime} / 3 F_{v}^{\prime \prime}$. If the Higgs potential only includes one higher-dimensional operator, say $\left(H^{\dagger} H\right)^{3}, \kappa_{3}$ is then proportional to its coefficient. In the general case with more than one higher-dimensional operator, $\kappa_{3}$ determines one of their linear combinations. For the later case, the quartic Higgs coupling receives new independent contribution from the fourth derivative of the potential, 
$\Gamma(h h h h)=\left.\frac{\partial^{4} V}{\partial h^{4}}\right|_{v}=3 F_{v}^{\prime \prime}+6 v^{2} F_{v}^{\prime \prime \prime}+v^{4} F_{v}^{(4)}=\frac{3 m_{h}^{2}}{v^{2}}\left(1+\kappa_{4}\right)$,

where $\kappa_{4} \equiv 2 v^{2} F_{v}^{\prime \prime \prime} / F_{v}^{\prime \prime}+v^{4} F_{v}^{(4)} / 3 F_{v}^{\prime \prime}$. As in (A4), the same combination $\kappa_{4}$ enters into the coupling for $h h h \pi^{0(+)} \pi^{0(-)}$. Such a relation can be generalized further when the potential has enough independent terms, namely, the self-coupling of $n$ Higgs is correlated with the coupling of a pair of Goldstone bosons and $(n-1)$ Higgs.

In summary, for the $\kappa_{4}$ dependence of the di-Higgs production cross section, we only need to consider the one-loop diagram from quartic Higgs coupling, while quartic couplings involving both Higgs and Goldstone are irrelevant.

\section{APPENDIX B: PERTURBATIVE UNITARITY BOUND}

To have the perturbative calculation still be reliable, Higgs self-couplings need to satisfy the perturbative unitarity bound. The scattering amplitude for $h h \rightarrow h h$ at tree level is

$\mathcal{M}(E, \theta)=-\lambda_{3}^{2}\left(\frac{1}{s-m_{h}^{2}}+\frac{1}{t-m_{h}^{2}}+\frac{1}{u-m_{h}^{2}}\right)-\lambda_{4}$

where the Mandelstam variables $s=E^{2}, \quad t=$ $-\left(E^{2}-4 m_{h}^{2}\right) \sin ^{2} \theta / 2$, and $u=-\left(E^{2}-4 m_{h}^{2}\right) \cos ^{2} \theta / 2$ in the center-of-mass frame. The partial wave amplitudes are then computed as $[45,46]$

$$
\begin{aligned}
a_{\ell}(E)= & \frac{1}{2} \frac{1}{32 \pi E^{2}} \beta\left(E^{2}, m_{h}^{2}, m_{h}^{2}\right) \\
& \times \int_{-1}^{1} \mathrm{~d} \cos \theta P_{\ell}(\cos \theta) \mathcal{M}(E, \theta),
\end{aligned}
$$

where the additional factor of $1 / 2$ comes from normalization of the symmetric initial, final states, and kinematic factor $\beta(x, y, z)=\left(x^{2}+y^{2}+z^{2}-2 x y-2 y z-2 x z\right)^{1 / 2}$. For the $\mathrm{s}$ wave, $\ell=0$, we find

$$
a_{0}(E)=-\frac{1}{32 \pi} \sqrt{\frac{E^{2}-4 m_{h}^{2}}{E^{2}}}\left[\lambda_{3}\left(\frac{1}{E^{2}-m_{h}^{2}}-\frac{2}{E^{2}-4 m_{h}^{2}} \log \frac{E^{2}-3 m_{h}^{2}}{m_{h}^{2}}\right)+\lambda_{4}\right] .
$$

The s-wave unitarity condition requires $\left|\operatorname{Re} a_{0}(E)\right|<1 / 2$. In the high-energy limit $E^{2} \gg m_{h}^{2}, \lambda_{4}$ contributes at the leading order. Thus, we can obtain $\left|\lambda_{4}\right|<16 \pi$, namely,

$$
\left|1+\kappa_{4}\right|<\frac{16 \pi v^{2}}{3 m_{h}^{2}}=65
$$

$\lambda_{3}$ starts to dominate at the low energy, and the amplitude reaches a peak at some scale. Assuming the peak amplitude satisfies the s-wave unitarity condition, we can find the range of $\kappa_{3}$ for a given $\kappa_{4}$ satisfying Eq. (B4).

\section{APPENDIX C: FIRST-ORDER ELECTROWEAK PHASE TRANSITION: A BENCHMARK}

The nature of EWPT could have a strong correlation with the Higgs potential at zero temperature. For illustrating the collider capability in probing the EWPT nature, we analyze a simplified model in the SMEFT [47] (we will tolerate the potential uncertainties caused by such a simplified treatment [48]; for discussions in more general contexts, see, e.g., Ref. [49]),

$$
\begin{aligned}
V_{\mathrm{SMEFT}}(T)= & \left(-\mu^{2}+a_{0} T^{2}\right) H^{\dagger} H+\lambda\left(H^{\dagger} H\right)^{2} \\
& +\frac{c_{6}}{\Lambda^{2}}\left(H^{\dagger} H\right)^{3}+\frac{c_{8}}{\Lambda^{4}}\left(H^{\dagger} H\right)^{4},
\end{aligned}
$$

as the benchmark. Here, the temperature-dependent term results from an expansion of thermal mass for the SM particles. $a_{0} \sim 3$ is defined by the SM physics. The firstorder EWPT requires the coexistence of two degenerate vacua, characterized by $v_{T}=0$ and $v_{T}=v_{c} \neq 0$ at the critical temperature $T_{c}$, with $v_{c}$ satisfying the following condition:

$$
\begin{gathered}
\left(v_{c}^{2}-v^{2}\right)^{2} v^{2}\left(3 \frac{c_{6}}{\Lambda^{2}}+4 \frac{c_{8}}{\Lambda^{4}} v^{2}+2 \frac{c_{8}}{\Lambda^{4}} v_{c}^{2}\right) \\
+2 m_{h}^{2}\left(v_{c}^{2}-v^{2}\right)+4 a_{0} T_{c}^{2} v^{2}=0 .
\end{gathered}
$$

Here, $m_{h}^{2}=2 \lambda v^{2}+\frac{3 c_{6} v^{4}}{\Lambda^{2}}+\frac{3 c_{8} v^{6}}{\Lambda^{4}}$ is the squared Higgs mass at zero temperature. We then scan over $\left\{c_{6}, c_{8}\right\}$, to extract out the $\left\{\kappa_{3}, \kappa_{4}\right\}$ region where a first-order EWPT is favored, using the relation

$$
\begin{aligned}
& \lambda_{3}=\frac{3 m_{h}^{2}}{v}\left(1+\frac{2 c_{6} v^{4}}{m_{h}^{2} \Lambda^{2}}+\frac{4 c_{8} v^{6}}{m_{h}^{2} \Lambda^{4}}\right) \\
& \lambda_{4}=\frac{3 m_{h}^{2}}{v^{2}}\left(1+\frac{12 c_{6} v^{4}}{m_{h}^{2} \Lambda^{2}}+\frac{32 c_{8} v^{6}}{m_{h}^{2} \Lambda^{4}}\right) .
\end{aligned}
$$

The favored region is marked in orange in Fig. 7. In the case with $c_{8}=0$, the orange region is reduced to the bottom boundary, which is consistent with the results obtained in Ref. [47], in which only the $\mathcal{O}_{6}$ operator is turned on. 


\section{APPENDIX D: $C_{3 a}$ AND $C_{4 b}$ FOR DI-HIGGS PRODUCTIONS AT ILC}

See Tables IV and V.

TABLE IV. The SM cross sections and the parametrization of the $\kappa_{3}, \kappa_{4}$ contributions in the di-Higgs invariant mass bins of $e^{-} e^{+} \rightarrow Z h h$ at ILC $500 \mathrm{GeV}$. Here, the ILC beam is polarized as $P\left(e^{-}, e^{+}\right)=(-0.8,0.3)$. Note that the $C_{3 a}$ and $C_{4 b}$ values are independent of the beam polarization.

\begin{tabular}{llllllc}
\hline \hline & $\sigma_{0}(\mathrm{fb})$ & $C_{31}$ & $C_{32}$ & $C_{41}$ & $C_{42}$ & $C_{43}$ \\
\hline$m_{h h}(\mathrm{GeV})$ & 0.232 & 0.564 & 0.0965 & -0.00517 & -0.0039 & -0.00081 \\
$(250,300)$ & 0.0647 & 0.862 & 0.195 & -0.00799 & -0.00771 & -0.00192 \\
$(300,350)$ & 0.0845 & 0.567 & 0.086 & -0.00516 & -0.00351 & -0.00061 \\
$(350,410)$ & 0.0826 & 0.328 & 0.03 & -0.00297 & -0.00131 & -0.00014 \\
\hline \hline
\end{tabular}

TABLE V. The SM cross sections and the parametrization of the $\kappa_{3}, \kappa_{4}$ contributions in the di-Higgs invariant mass bins of $e^{-} e^{+} \rightarrow$ $\nu \bar{\nu} h h$ at ILC $1 \mathrm{TeV}$. The ILC beam is polarized as $P\left(e^{-}, e^{+}\right)=(-0.8,0.2)$. Note that the $C_{3 a}$ and $C_{4 b}$ values are independent of the beam polarization.

\begin{tabular}{llllllr}
\hline \hline & $\sigma_{0}(\mathrm{fb})$ & $C_{31}$ & $C_{32}$ & $C_{41}$ & $C_{42}$ & $C_{43}$ \\
\hline$m_{h h}(\mathrm{GeV})$ & 0.159 & -1.20 & 1.10 & -0.00327 & 0.0079 & -0.0075 \\
$(250,350)$ & 0.0458 & -1.96 & 2.62 & -0.00642 & 0.0189 & -0.0226 \\
$(350,450)$ & 0.0540 & -1.20 & 0.741 & -0.00321 & 0.00642 & -0.00301 \\
$(450,550)$ & 0.0344 & -0.744 & 0.322 & -0.0014 & 0.00167 & -0.000245 \\
$(550,650)$ & 0.0168 & -0.513 & 0.180 & -0.000446 & -0.000013 & 0.000303 \\
$(650,750)$ & 0.00649 & -0.376 & 0.114 & 0.0000953 & -0.000682 & 0.000412 \\
$(750,850)$ & 0.00178 & -0.281 & 0.0768 & 0.000402 & -0.000921 & 0.000397 \\
$(850,1000)$ & 0.000242 & -0.198 & 0.0501 & 0.000526 & -0.000897 & 0.000323 \\
\hline \hline
\end{tabular}

[1] ATLAS Collaboration, Study of the double Higgs production channel $H(\rightarrow b \bar{b}) H(\rightarrow \gamma \gamma)$ with the ATLAS experiment at the HL-LHC, CERN Report No. ATL-PHYS-PUB-2017001.

[2] J. H. Kim, Y. Sakaki, and M. Son, Phys. Rev. D 98, 015016 (2018).

[3] W. Yao, arXiv:1308.6302.

[4] F. Goertz, A. Papaefstathiou, L. L. Yang, and J. Zurita, J. High Energy Phys. 06 (2013) 016.

[5] D. Goncalves, T. Han, F. Kling, T. Plehn, and M. Takeuchi, Phys. Rev. D 97, 113004 (2018).

[6] H. J. He, J. Ren, and W. Yao, Phys. Rev. D 93, 015003 (2016).

[7] R. Contino et al., CERN Yellow Rep. 3, 255 (2017).

[8] M. J. Dolan, C. Englert, N. Greiner, K. Nordstrom, and M. Spannowsky, Eur. Phys. J. C 75, 387 (2015).
[9] F. Bishara, R. Contino, and J. Rojo, Eur. Phys. J. C 77, 481 (2017).

[10] C. Englert, F. Krauss, M. Spannowsky, and J. Thompson, Phys. Lett. B 743, 93 (2015).

[11] T. Liu and H. Zhang, arXiv:1410.1855.

[12] Q. H. Cao, Y. Liu, and B. Yan, Phys. Rev. D 95, 073006 (2017).

[13] D. M. Asner et al., arXiv:1310.0763.

[14] H. Abramowicz et al., Eur. Phys. J. C 77, 475 (2017).

[15] T. Plehn and M. Rauch, Phys. Rev. D 72, 053008 (2005).

[16] A. Papaefstathiou and K. Sakurai, J. High Energy Phys. 02 (2016) 006.

[17] C. Y. Chen, Q. S. Yan, X. Zhao, Y. M. Zhong, and Z. Zhao, Phys. Rev. D 93, 013007 (2016).

[18] B. Fuks, J. H. Kim, and S. J. Lee, Phys. Rev. D 93, 035026 (2016). 
[19] W. Kilian, S. Sun, Q. S. Yan, X. Zhao, and Z. Zhao, J. High Energy Phys. 06 (2017) 145.

[20] G. Degrassi, P. P. Giardino, F. Maltoni, and D. Pagani, J. High Energy Phys. 12 (2016) 080.

[21] W. Bizon, M. Gorbahn, U. Haisch, and G. Zanderighi, J. High Energy Phys. 07 (2017) 083.

[22] I. Brivio and M. Trott, arXiv:1706.08945.

[23] G. Panico and A. Wulzer, Lect. Notes Phys. 913, 1 (2016).

[24] E. Halyo, Mod. Phys. Lett. A 08, 275 (1993).

[25] W. D. Goldberger, B. Grinstein, and W. Skiba, Phys. Rev. Lett. 100, 111802 (2008).

[26] G. Buchalla, O. Cata, A. Celis, and C. Krause, Nucl. Phys. B917, 209 (2017).

[27] V. Barger, T. Han, P. Langacker, B. McElrath, and P. Zerwas, Phys. Rev. D 67, 115001 (2003).

[28] M. McCullough, Phys. Rev. D 90, 015001 (2014); 92, 039903(E) (2015).

[29] A. Alloul, N. D. Christensen, C. Degrande, C. Duhr, and B. Fuks, Comput. Phys. Commun. 185, 2250 (2014).

[30] T. Hahn, Comput. Phys. Commun. 140, 418 (2001).

[31] T. Hahn and M. Perez-Victoria, Comput. Phys. Commun. 118, 153 (1999).

[32] C. Patrignani et al. (Particle Data Group), Chin. Phys. C 40, 100001 (2016).

[33] J. Alwall, R. Frederix, S. Frixione, V. Hirschi, F. Maltoni, O. Mattelaer, H.-S. Shao, T. Stelzer, P. Torrielli, and M. Zaro, J. High Energy Phys. 07 (2014) 079.

[34] A. Belyaev, N. D. Christensen, and A. Pukhov, Comput. Phys. Commun. 184, 1729 (2013).
[35] J. Baglio, A. Djouadi, R. Grber, M. M. Mhlleitner, J. Quevillon, and M. Spira, J. High Energy Phys. 04 (2013) 151.

[36] R. Frederix, S. Frixione, V. Hirschi, F. Maltoni, O. Mattelaer, P. Torrielli, E. Vryonidou, and M. Zaro, Phys. Lett. B 732, 142 (2014).

[37] T. Barklow, J. Brau, K. Fujii, J. Gao, J. List, N. Walker, and K. Yokoya, arXiv:1506.07830.

[38] M. J. Boland et al. (CLIC and CLICdp Collaborations), arXiv:1608.07537.

[39] S. Di Vita, G. Durieux, C. Grojean, J. Gu, Z. Liu, G. Panico, M. Riembau, and T. Vantalon, J. High Energy Phys. 02 (2018) 178.

[40] J. Ellis and T. You, J. High Energy Phys. 03 (2016) 089.

[41] G. Durieux, C. Grojean, J. Gu, and K. Wang, J. High Energy Phys. 09 (2017) 014.

[42] T. Barklow, K. Fujii, S. Jung, R. Karl, J. List, T. Ogawa, M. E. Peskin, and J. Tian, Phys. Rev. D 97, 053003 (2018).

[43] W. H. Chiu, S. C. Leung, T. Liu, K. F. Lyu, and L. T. Wang, J. High Energy Phys. 05 (2018) 081.

[44] F. Maltoni, D. Pagani, and X. Zhao, J. High Energy Phys. 07 (2018) 087.

[45] L. Di Luzio, J. F. Kamenik, and M. Nardecchia, Eur. Phys. J. C 77, 30 (2017).

[46] L. Di Luzio, R. Grber, and M. Spannowsky, Eur. Phys. J. C 77, 788 (2017).

[47] P. Huang, A. Joglekar, B. Li, and C. E. M. Wagner, Phys. Rev. D 93, 055049 (2016).

[48] M. Chala, C. Krause, and G. Nardini, J. High Energy Phys. 07 (2018) 062.

[49] B. Jain, S. J. Lee, and M. Son, Phys. Rev. D 98, 075002 (2018). 